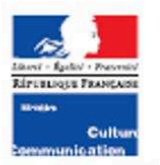

Secretarlat genera

Service de la

coordination des

polltiques cultureles

et de rinnovation

Departement
des etudes,

de la prospective
et des statistiques

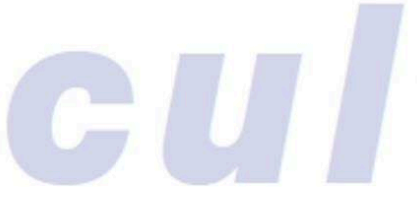

82, rue Saint-Honoré, 75033 Paris cedex 01

\& 0140157917 - ๑ 0140157999

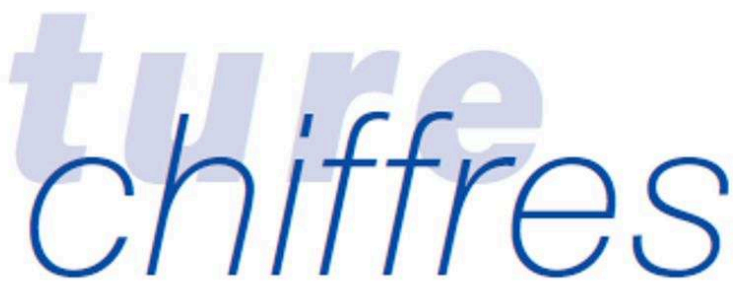

\title{
Diversité des carrières d'auteur de l'audiovisuel et du spectacle vivant. Les auteurs de la SACD, 1997-2008
}

Marie GouYon*

Diverse careers amongst audiovisual and live entertainment authors. SACD Authors, 1997-2008

\begin{tabular}{|c|c|c|c|}
\hline \multicolumn{4}{|c|}{ Sommaire } \\
\hline Une vue d'ensemble. & 2 & $5 \%$ des auteurs concentrent $28 \%$ des contributions & \\
\hline $\begin{array}{l}\text { L'audiovisuel et le spectacle vivant : } \\
\text { deux répertoires très distincts ........ }\end{array}$ & & $\begin{array}{l}\text { à la création et } 67 \% \text { des droits ........................................................................ } \\
\text { Des disparités selon le seeve }\end{array}$ & $\begin{array}{l}11 \\
11\end{array}$ \\
\hline $\begin{array}{l}\text { deux repertoires trés distincts ............... } \\
330000 \text { créations audiovisuelles sont répertorićes }\end{array}$ & 4 & $\begin{array}{l}\text { Les plus de } 50 \text { ans représentent une part croissante } \\
\text { des auteurs du spectacle vivant }\end{array}$ & \\
\hline La diffusion des ceuvres audiovisuelles s'est fortement & & La majorité des auteurs vivent bors de I'lle-de-France ....... & 11 \\
\hline 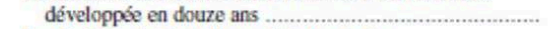 & 4 & Les sociétaires représentent $17 \%$ des auteurs & \\
\hline $\begin{array}{l}\text { Les fictions s'attirent l'essentiel des droits } \\
\text { mais représentent moins de la moitié des créations ......... }\end{array}$ & 5 & $\begin{array}{l}\text { du spectacle vivant et } 55 \% \text { des droits engendrés } \\
\text { par oe répertoire }\end{array}$ & 14 \\
\hline $\begin{array}{l}5 \% \text { des auteurs concentrent } 67 \% \text { des contributions } \\
\text { à la diffusion audiovisuelle et } 50 \% \text { des revenus ............. }\end{array}$ & 6 & Une typologie des carrières d'auteurs & \\
\hline $\begin{array}{l}\text { Les femmes représentent un quart des auteurs, des droits } \\
\text { et des contributions à la création et à la diffusion ......... }\end{array}$ & 6 & Type I : Les createurs actifs du spectacle vivant (18 \%) ..... & $\begin{array}{l}15 \\
16\end{array}$ \\
\hline Les fernmes créent le plus souvent des fictions & & Type II : Les auteurs en retrait du spectacle vivant $(17 \%)$.. & 18 \\
\hline télóvisuelles, tandis que les hommes se concentrent & & Type III : Les créateurs actifs de l'audiovisuel ( $10 \%)$........ & 20 \\
\hline davantage sur les fictions cinématographiques ...... & 6 & Type IV : La relève $\alpha$ animśe $*(6 \%) \ldots \ldots \ldots \ldots \ldots$ & 22 \\
\hline $\begin{array}{l}\text { Les auteurs de l'audiovisuel ont en moyenne } 49 \text { ans } \\
\text { en } 2008\end{array}$ & 7 & $\begin{array}{l}\text { Type V : La relève cinématographique }(18 \%) \\
\text { Type VI : Les éphémères }(31 \%)\end{array}$ & $\begin{array}{l}24 \\
26\end{array}$ \\
\hline $\begin{array}{l}\text { Plus de } 80 \% \text { des auteurs vivent en France, } \\
\text { dont } 80 \% \text { en région parisienne }\end{array}$ & 7 & 1уре & \\
\hline $\begin{array}{l}\text { Les sociétaires représentent } 30 \% \text { des auteurs de l'audiowisuel } \\
\text { et se partagent } 68 \% \text { des droits dans ce répertoire }\end{array}$ & 9 & Encadré 1 - La sucs: rôle et fonctionnement .......... & 2 \\
\hline $\begin{array}{l}\text { Près de } 10000 \text { auteurs interviennent } \\
\text { dans le spectacle vivant en } 2008\end{array}$ & S & compositeurs dramatiques entre 1997 et 2008 ................ & 15 \\
\hline Le nombre de créateurs du spectacle vivant & & $\begin{array}{l}\text { Encadré 4 - Caractéristiques distinctives des carrières types : } \\
\text { représentations graphiques }\end{array}$ & 28 \\
\hline 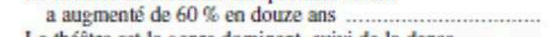 & 9 & Méthodologie - Les donées transmises par la SACD & \\
\hline Le theatre est le genre dominant, suivi de la danse . & 10 & 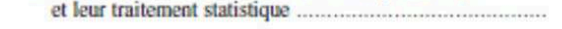 & 32 \\
\hline
\end{tabular}

* Attachée statisticienne de l'Insee, chargée d'analyse au dẹps.

Cette étude est le fruit d'un partenariat entre la sAcD et le press, permettant à ce demier d'exploiter statistiquement les données recueillies par la sMop. Doivent en particulier être remerciés : Pascal Rocinz, directeur général de la sucD, Debora Asrussowicz, directrioe des projets de développements éditoriaux, Jérôme Dechrssn, directeur adjoint de l'audiovisuel, Linda ConvinlıH, directrice du spectacle vivant, Muriel Couson, directrioe du dévelop-

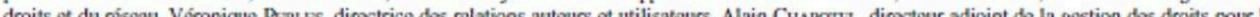
pốle estion collectiv del' 


\section{Portrait économique des diffuseurs d'art actuel inscrits à la Maison des artistes}

Economic picture of contemporary art dealers registered with the Maison des Artistes

Muriel de Vrièse, Bénédicte Martin, Nathalie Moureau et Dominique SagotDuvauroux

Éditeur : Département des études, de la prospective et des statistiques

Lieu d'édition : Paris

Année d'édition : 2011

Date de mise en ligne : 21 septembre 2015

Collection : Culture chiffres

ISBN électronique : 9782111398535

\section{C books}

http://books.openedition.org

\section{Édition imprimée}

Date de publication : 1 janvier 2011

Nombre de pages : 8

\section{Référence électronique}

VRIĖSE, Muriel de ; et al. Portrait économique des diffuseurs d'art actuel inscrits à la Maison des artistes. Nouvelle édition [en ligne]. Paris : Département des études, de la prospective et des statistiques, 2011 (généré le 25 avril 2021). Disponible sur Internet : <http://books.openedition.org/deps/541>. ISBN 9782111398535 

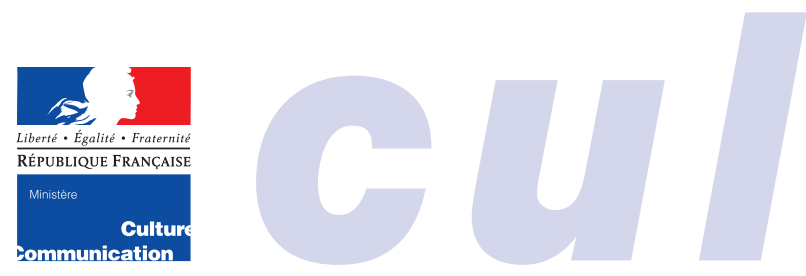

Secrétariat général

Service de la

coordination des

politiques culturelles

et de l'innovation

Département

des études,

de la prospective

et des statistiques

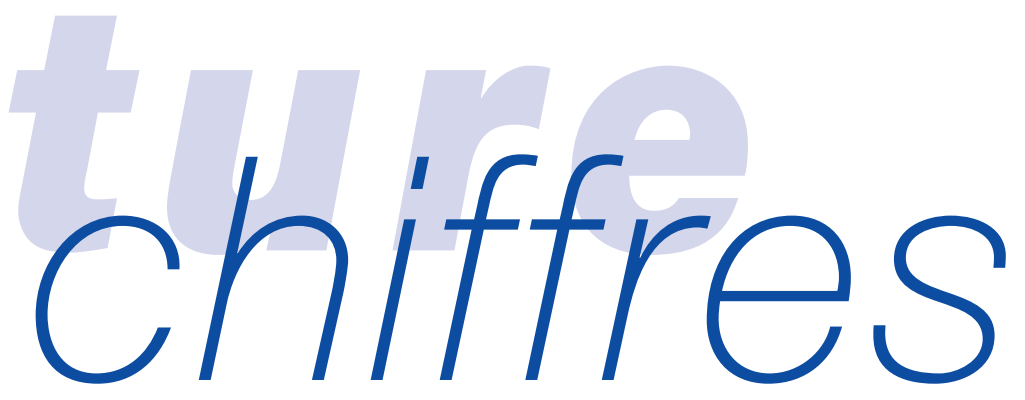

\title{
Portrait économique des diffuseurs d'art actuel inscrits à la Maison des artistes
}

\author{
Muriel de Vrièse, Bénédicte MArtin, Nathalie Moureau, Dominique Sagot-Duvauroux* \\ Economic picture of contemporary art dealers \\ registered with the Maison des Artistes
}

Les diffuseurs français d'art actuel demeurent aujourd'hui encore mal connus. En raison de l'absence de code APE spécifique pour les recenser, pratiquement aucune donnée statistique n'est produite les concernant et peu d'études empiriques leur sont consacrées. L'analyse des déclarations à la Maison des artistes - organisme chargé de gérer le régime de sécurité sociale de ces professionnels - d'un ensemble représentatif de diffuseurs qui exposent et vendent les œuvres d'artistes vivants (voir l'encadré méthodologie en p. 8) permet de dégager des informations inédites et de caractériser la nature des relations qu'ils entretiennent avec les artistes qu'ils soutiennent.

\section{Portrait des diffuseurs D'ART ACTUEL}

\section{Plutôt jeunes, inégalement répartis sur le territoire et essentiellement des galeries}

Les diffuseurs d'art actuel sont très majoritairement des galeries $(92 \%)$, une part réduite exerce à titre principal le métier d'antiquaire ou de brocanteur (6\%), tandis que les éditeurs d'art et les musées ne représentent qu'une très faible proportion $(2 \%)$.
Dans l'ensemble, ces structures sont relativement jeunes : en 2006, les trois quarts de ces structures ont été créées il y a moins de vingt ans, une structure sur cinq a moins de six ans d'exercice. La moyenne d'âge, 15 ans, demeure peu élevée. $5 \%$ seulement des diffuseurs existent depuis plus de 40 ans.

On trouve peu d'associations au sein de la population des diffuseurs $(5 \%)$, qui optent de préférence pour un statut de société à responsabilité limitée (SARL) $(47 \%)$ et d'entreprise patrimoniale (EP) (35\%). Les entreprises unipersonnelles à responsabilité limitée (EURL) (4\%), ainsi que les sociétés en nom collectif (SNC) et les sociétés par actions simplifiées (SAS) sont moins bien représentées (moins de $1 \%$ ).

Les diffuseurs d'art actuel sont très inégalement répartis sur le territoire français : $42 \%$ sont concentrés en Île-deFrance (dont $87 \%$ à Paris) ; les autres se localisent en Provence-Alpes-Côte d'Azur (14\%), en Rhône-Alpes (7\%), en Bretagne $(5 \%)$ et en Aquitaine $\left(5 \%^{1}\right)$.

\section{Évaluation économique des liens entre artistes et diffuseurs}

En 2006, parmi les diffuseurs d'art actuel - qui vendent des œuvres d'artistes français (résidant en France) vivants -, $78 \%$ sont concentrés exclusivement sur cette activité, les autres l'ont complétée par la vente d'œuvres d'artistes décédés.

\footnotetext{
* Muriel de VRIÈSE, Université du Havre. Bénédicte Martin, Université du Havre. Nathalie Moureau, Laboratoire montpelliérain d'économie théorique et appliquée (Lameta), Université de Montpellier. Dominique SAGOT-DuvAUROUX, Groupe de recherche angevin en économie et management (Granem), Université d'Angers.

1. Un tiers des régions n'accueille qu'une proportion marginale de ces diffuseurs d'art actuel français : Alsace, Antilles Guyane Réunion, Auvergne, Centre, Champagne-Ardenne, Corse, Franche-Comté, Limousin, Lorraine, Picardie, Poitou-Charentes. Le faible effectif des structures de ces onze régions ne permet pas de les traiter isolément : dans la suite du texte, elles seront regroupées dans une catégorie « Autres régions ». Paris, en revanche, est isolé du reste de la région Île-de-France en raison de son caractère particulier.
} 
Les diffuseurs versent en moyenne 82800 euros par an, cette somme se répartissant sur dix artistes. L'éventail des sommes distribuées est très large : de 72 euros pour la plus faible à 487200 euros par artiste (soit un écart type de 33300 euros). La moyenne pondérée des sommes versées par artiste est de 12000 euros $^{2}$.

Le phénomène de polarisation sur les artistes locaux semble très fort puisque plus de la moitié des artistes rémunérés résident dans la même région que leur diffuseur (soit $52 \%$ ). Par ailleurs, $83 \%$ des diffuseurs versent des sommes à au moins un artiste français vivant dans leur région (et $72 \%$ dans leur département). Cette proportion augmente de $7 \%$ entre 2004 et 2006.

Au-delà de ce phénomène de polarité locale, les diffuseurs semblent s'appuyer sur le travail d'un noyau dur d'artistes dont les œuvres se vendent régulièrement (38\% de leurs artistes en moyenne). Pour la plupart des diffuseurs (59\%), ce noyau dur regroupe un à cinq artistes (graphique 1). Plus largement, plus de $80 \%$ des diffuseurs versent des sommes à au moins un artiste « régulier $^{3} »$.

\section{LE CHIFFRE D'AFFAIRES \\ DES DIFFUSEURS D'ART ACTUEL ET SES DÉTERMINANTS}

Le chiffre d'affaires annuel ${ }^{4}$ moyen par diffuseur s'élève à 404700 euros entre 2004 et 2006. Cette moyenne cache une hétérogénéité très forte: de 0 à 14046900 euros selon les structures. Avec une progression de plus d'un quart $(28 \%)$ entre 2004 et 2006, principalement en $2006(+22 \%)$, le premier marché semble donc avoir profité de la hausse générale des prix sur le marché de l'art et de l'engouement pour l'art contemporain sensible sur le marché des enchères ${ }^{5}$. Pour autant, une grande proportion de diffuseurs d'art actuel réalise un chiffre d'affaires qui reste faible (43\% ont un chiffre d'affaires inférieur à 50000 euros) (graphique 2).

\section{Localisation}

Le chiffre d'affaires des diffuseurs dépend de leur implantation territoriale: le chiffre d'affaires moyen des structures parisiennes s'élève à 818500 euros contre une moyenne de 146200 euros pour toutes les autres structures.

\section{Structure juridique}

Statut juridique et chiffre d'affaires sont significativement liés. Le fait d'être une association ou une entreprise patrimoniale est lié négativement au résultat économique, contrairement au fait d'être une SA ou une SARL. Ainsi, les diffuseurs dont le chiffre d'affaires moyen est le plus élevé sont davantage constitués en SAS et SA (respectivement 1,9 million d'euros et 1,6 million d'euros en moyenne). Les SARL (482 000 euros de chiffre d'affaires annuel moyen) et les SNC

\section{Graphique 1 - Répartition des diffuseurs en fonction du nombre d'artistes "réguliers" auxquels ils versent des sommes}

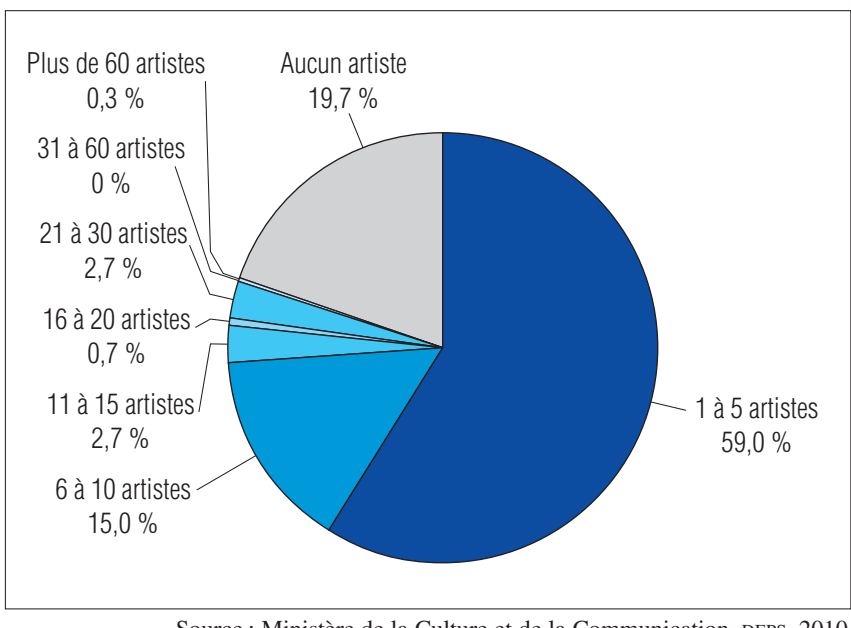

\section{Graphique 2 - Répartition des diffuseurs par tranches de chiffre d'affaires annuel (moyenne sur trois ans)}

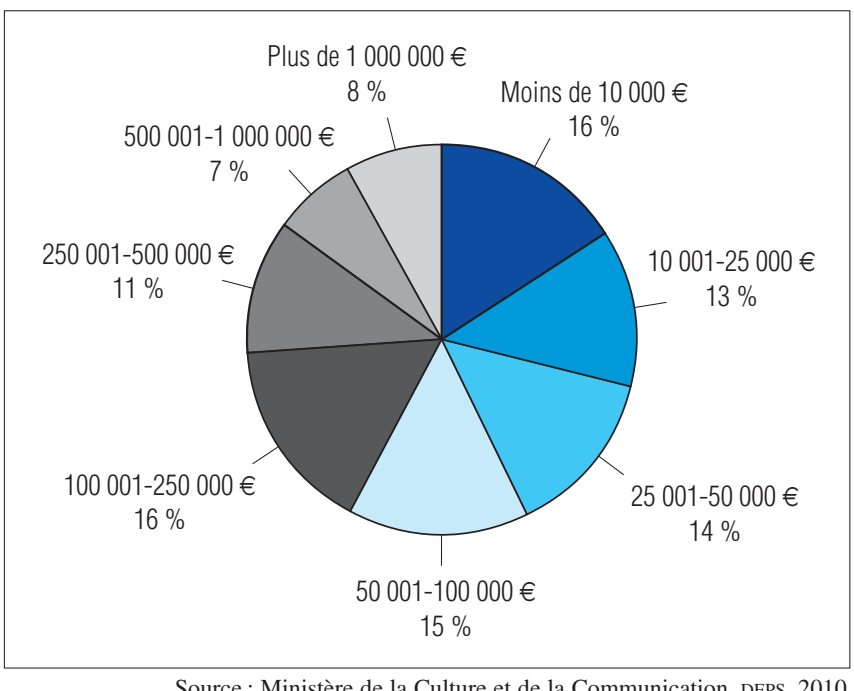

(421000 euros) ont un chiffre d'affaires moyen plus faible. Viennent ensuite les EURL (186000 euros), les entreprises patrimoniales (99000 euros) et enfin les associations (62 500 euros).

\section{Activité}

L'analyse du chiffre d'affaires des diffuseurs inscrits à la Maison des artistes révèle la diversité des situations économiques : certaines activités comme l'édition d'art sont plus lucratives que d'autres (les brocantes), tandis que les structures les plus jeunes, comme les TPE (très petites entreprises),

2. Ces sommes représentent en moyenne $55 \%$ du chiffre d'affaires annuel des structures. Ce ratio peut parfois être supérieur à $100 \%$, lorsque les structures ne rémunèrent pas les artistes lors de l'année fiscale en cours, créant ainsi des décalages dans le temps.

3. Ces proportions sont calculées sur la base de la population totale des structures de diffusion ayant déclaré le nom des artistes auxquels elles ont versé des sommes, soit 300 structures chaque année.

4. Il s'agit du chiffre d'affaires total TTC du diffuseur au titre de l'ensemble de ses activités.

5. Selon l'Artprice Global Index, les prix sur le marché des enchères ont augmenté de $37 \%$ entre le $1^{\text {er }}$ janvier 2004 et le $1^{\text {er }}$ janvier 2007 ; les prix aux enchères d'œuvres d'art contemporain ont quant à eux augmenté de $72 \%$ durant la même période. 


\section{Méthodologie de l'analyse économétrique du chiffre d'affaires des diffuseurs}

Une analyse économétrique a été réalisée pour apprécier l'influence d'une série de facteurs sur l'activité économique des diffuseurs d'art actuel :

- la forme juridique du diffuseur : EP, SARL, SA, ASS, EURL et les autres formes juridiques (SAS et SNS) ont été regroupées dans une même catégorie du fait d'un trop petit nombre d'observations ;

- l'ancienneté de la structure : de 0 à 5 ans, de 6 à 10 ans, de 11 à 20 ans, de 21 à 30 ans, de 31 à 40 ans et plus de 40 ans ;

- la localisation géographique : Aquitaine, Basse-Normandie, Bourgogne, Languedoc-Roussillon, Midi-Pyrénées, Nord-Pasde-Calais, Pays de la Loire, Haute-Normandie, PACA, Rhône-Alpes, Bretagne, Paris, petite et grande couronne et les autres régions ;

- le fait que le diffuseur vende ou non, à côté d'œuvres d'artistes français vivants, des œuvres d'artistes français décédés ;

- le nombre d'artistes français vivants à qui la structure de diffusion verse des sommes lors de l'année fiscale en cours ;

- la somme versée par artiste français vivant;

- la proportion d'artistes régionaux auxquels la structure verse les sommes lors de l'année fiscale en cours ;

- la proportion d'artistes « réguliers » à qui la structure verse des sommes lors de l'année fiscale en cours.

souffrent d'une espérance de vie faible et d'une grande fragilité financière. L'activité d'édition d'art est celle dont le résultat économique est le plus élevé (chiffre d'affaires annuel moyen de 469400 euros). À l'inverse, les brocanteurs (21 400 euros) et les antiquaires (152 200 euros) réalisent les chiffres d'affaires annuels moyens les plus faibles. Les galeries (398 000 euros) sont les structures les plus nombreuses, et donc les mieux réparties entre les tranches de chiffre d'affaires.

\section{Âge}

Paradoxalement, l'ancienneté de la structure n'a que peu d'influence sur le chiffre d'affaires au-delà du seuil de cinq années d'activités. Le chiffre d'affaires moyen des structures de moins de cinq ans (94000 euros) est dix fois moins élevé que celui des structures âgées de 31 à 40 ans (949 000 euros). Plus de $43 \%$ des structures de moins de cinq ans ont un chiffre d'affaires inférieur à 25000 euros, et $40 \%$ des structures de plus de 40 ans un chiffre d'affaires supérieur à 500000 euros.

\section{Valorisation des artistes vivants et spécialisation de l'activité}

Se consacrer à la vente exclusive d'œuvres d'artistes français vivants est économiquement moins porteur que d'intervenir également sur le marché des artistes décédés: une grande proportion des diffuseurs dont le travail est consacré exclusivement à la vente d'œuvres d'artistes vivants réalisent un chiffre d'affaires faible ( $41 \%$ d'entre eux ont un chiffre d'affaires inférieur à 50000 euros), contrairement aux diffuseurs qui vendent à la fois les œuvres d'artistes vivants et d'artistes décédés (26\% déclarent un chiffre d'affaires supérieur à 500000 euros), et à ceux qui ne vendent que les œuvres d'artistes décédés (30\% ont un chiffre d'affaires supérieur à 500000 euros). Ainsi la répartition des risques entre artistes vivants et artistes décédés reste-t-elle un gage de pérennité et d'efficacité économique.

Si le chiffre d'affaires des diffuseurs tend à évoluer, faiblement, à l'inverse du nombre d'artistes rémunérés, on observe un effet de seuil : le nombre d'artistes qui vendent augmente progressivement avec le chiffre d'affaires des dif- fuseurs jusqu'à un million d'euros puis décroît ; les structures les mieux dotées économiquement ne sont donc pas celles qui rémunèrent le plus grand nombre d'artistes.

En revanche, les sommes versées aux artistes sont liées positivement au chiffre d'affaires. Si les structures les plus importantes ne sont donc pas celles qui rémunèrent le plus grand nombre d'artistes, ce sont celles qui versent les sommes les plus élevées par artiste : 48300 euros en moyenne par artiste pour les structures dont le chiffre d'affaires est supérieur à un million d'euros, signe qu'elles prennent en charge des artistes bénéficiant d'une certaine notoriété.

Le chiffre d'affaires des diffuseurs semble faiblement corrélé à la proportion d'artistes de leur région. Cette proportion s'élève à $58 \%$ pour les structures qui déclarent un chiffre d'affaires annuel très faible (inférieur à 10000 euros), contre $56 \%$ pour celles qui déclarent un chiffre d'affaires compris entre 250001 et 500000 euros et $50 \%$ pour celles qui déclarent un chiffre d'affaires élevé (supérieur à 1 million d'euros). Si l'on peut supposer que le fait de privilégier les artistes locaux permet de se constituer un marché, on ne saurait en déduire que c'est une stratégie efficace pour développer le chiffre d'affaires.

En revanche, le chiffre d'affaires est positivement corrélé à la proportion d'artistes réguliers (dont les œuvres sont vendues de façon régulière par les diffuseurs) : $52 \%$ des diffuseurs déclarant un chiffre d'affaires supérieur à un million d'euros font régulièrement affaire avec les mêmes artistes contre $29 \%$ des diffuseurs déclarant un chiffre d'affaires inférieur à 10000 euros. Le volume d'affaires d'un diffuseur est donc lié à sa capacité à s'appuyer sur un noyau d'artistes dont il vend régulièrement les œuvres, ce qui incite les diffuseurs à entretenir des relations durables avec les artistes auxquels ils ont su trouver un marché. Cela peut aboutir à deux stratégies opposées : une stratégie de neutralisation du risque - cette base d'artistes réguliers facilite la valorisation du travail de nouveaux artistes - ou, au contraire, une stratégie d'investissement sur quelques artistes réguliers qui conduit le diffuseur à limiter son travail de valorisation à un petit nombre d'artistes, au détriment d'autres, plus risqués.

Stratégiquement, les diffuseurs ont tout intérêt à constituer un noyau d'artistes réguliers suffisamment large pour consolider leur chiffre d'affaires. L'une des plus grandes fractures entre les diffuseurs demeure celle de la localisation géographique. 


\section{SPÉCIFICITÉS DES DIFFUSEURS PARISIENS}

Au-delà de la concentration géographique parisienne ${ }^{6}$ des structures de diffusion, on observe une inégale répartition du chiffre d'affaires sur le territoire : $78 \%$ du chiffre d'affaires total de l'activité de diffusion est réalisé par les structures parisiennes. De plus, la progression du chiffre d'affaires observée entre 2004 et 2006 est surtout due à l'activité des structures parisiennes $(+40 \%$, contre $+7 \%$ hors Paris).

\section{Les diffuseurs parisiens}

La forte concentration géographique des diffuseurs dans Paris $(87 \%$ des diffuseurs d'Île-de-France sont implantés à Paris, $6 \%$ en petite couronne et $7 \%$ en grande couronne) cache une hétérogénéité de situations importante, que ce soit en termes de localisation géographique, d'activités ou de spécialisation des structures. Une analyse par quartier permet de mettre au jour ces disparités.

À l'intérieur de Paris, les quartiers Est ( $9 \%$ ) sont très largement délaissés au profit de la Rive gauche (38\%, essentiellement dans le $6^{\mathrm{e}}$ arrondissement), de la rive droite Beaubourg-Marais (31\%, surtout dans le $3^{\mathrm{e}}$ arrondissement) et de la rive droite Matignon (22\%, essentiellement dans le $8^{\mathrm{e}}$ arrondissement $^{7}$ ) (tableau 1). De même, la vigueur économique des diffuseurs diffère fortement d'un quartier à l' autre : le chiffre d'affaires annuel moyen le plus élevé est réalisé par les diffuseurs de la Rive droite - soit 1,3 million d'euros dans le quartier de Matignon et 890000 euros dans le quartier Beaubourg-Marais -, quand les diffuseurs de la Rive gauche réalisent un chiffre d'affaires annuel moyen de 600000 euros (tableau 1).

Plusieurs facteurs expliquent cette hétérogénéité de situations. D'abord, la corrélation existant entre l'âge de la structure et son quartier d'implantation ${ }^{8}$ : plus d'un tiers des structures du quartier Est existent depuis moins de dix ans, alors qu'environ un tiers de celles du quartier Matignon comptent plus de trente ans d'activité. Les quartiers Beaubourg-Marais (43\% ont entre 11 et 20 ans) et de la Rive gauche (37\%) occupent une position intermédiaire.

Ensuite, la spécialisation des structures par quartier est manifeste. Comparativement aux autres régions, c'est à Paris que les structures ont le plus recours à la vente d'œuvres d'artistes décédés, en particulier dans le quartier Matignon. À l'inverse, dans les quartiers Est, plus jeunes, les structures privilégient la vente exclusive d'œuvres d'artistes vivants (81\%). Ce phénomène de spécialisation ressort également de l'observation du nombre moyen d'artistes français vivants rémunérés par les structures : celui-ci est beaucoup plus faible pour les structures du quartier Matignon (2,7) que pour celles de la Rive gauche $(6,4)$, du quartier Beaubourg-Marais $(7,8)$ ou encore des quartiers Est $(14,8)$.

De plus, Paris profite de la concentration d'artistes qui y résident puisque c'est le territoire le plus marqué par le phénomène $\mathrm{du}$ «localisme » $(63 \%$ des artistes français vivants rémunérés par les diffuseurs vivent en région parisienne). La proportion d'artistes locaux est plus importante dans les structures des quartiers Est (77\%) que dans le quartier de Beaubourg-Marais (69\%), de la Rive gauche (62\%); elle est moins élevée dans le quartier Matignon (52\%).

La proportion d'artistes réguliers des structures parisiennes est importante : $45 \%$ dans les quartiers Est et $42 \%$ dans les quartiers Beaubourg-Marais ainsi que dans ceux de la Rive gauche ; elle est en revanche moins élevée pour les diffuseurs du quartier Matignon (32\%). Être une structure plus ancienne ne semble donc pas influer significativement sur la constitution d'un noyau d'artistes plus large, qui augmenterait avec le temps. Cependant, les structures conservant la plus grande proportion d'artistes réguliers sont, pour chaque quartier, celles qui ont un chiffre d'affaires élevé (hormis les quelques structures de la Rive gauche qui ont un chiffre d'affaires inférieur à 10000 euros). La proportion d'artistes réguliers est donc positivement corrélée au niveau de chiffre d'affaires de manière plus significative pour les structures de diffusion parisiennes que pour les autres.

\section{Tableau 1 - Caractéristiques des diffuseurs parisiens par quartier (moyennes annuelles sur la période 2006-2008)}

\begin{tabular}{|c|c|c|c|c|c|c|c|c|c|}
\hline \multirow[t]{2}{*}{ Quartiers } & \multirow{2}{*}{$\begin{array}{l}\text { Répartition } \\
\text { géographique }\end{array}$} & \multirow{2}{*}{$\begin{array}{c}\text { CA } \\
\text { moyen } \\
(e n €)\end{array}$} & \multirow{2}{*}{$\begin{array}{c}\text { Âge } \\
\text { moyen }\end{array}$} & \multirow{2}{*}{$\begin{array}{c}\text { Nombre } \\
\text { d'artistes } \\
\text { français } \\
\text { vivants }\end{array}$} & \multicolumn{3}{|c|}{ Structures qui rémunèrent des artistes } & \multicolumn{2}{|c|}{ Proportion d'artistes } \\
\hline & & & & & vivants & $\begin{array}{l}\text { vivants } \\
\text { et décédés }\end{array}$ & décédés & locaux & réguliers \\
\hline $\begin{array}{l}\text { Rive gauche } \\
\qquad\left(5^{\mathrm{e}}, 6^{\mathrm{e}}, 7^{\mathrm{e}}, 14^{\mathrm{e}}\right)\end{array}$ & 38 & 600000 & 22,1 & 6,4 & 65 & 32 & 4 & 62 & 42 \\
\hline $\begin{array}{l}\text { Rive droite Beaubourg- } \\
\text { Marais }\left(1^{\mathrm{er}}, 2^{\mathrm{e}}, 3^{\mathrm{e}}, 4^{\mathrm{e}}\right)\end{array}$ & 31 & 890000 & 16,4 & 7.8 & 75 & 22 & 3 & 69 & 42 \\
\hline $\begin{array}{l}\text { Rive droite Matignon } \\
\qquad\left(8^{\mathrm{e}}, 9^{\mathrm{e}}, 16^{\mathrm{e}}, 17^{\mathrm{e}}\right)\end{array}$ & 22 & 1300000 & 20,5 & 2,7 & 49 & 43 & 8 & 52 & 32 \\
\hline $\begin{array}{l}\text { Quartiers Est }\left(10^{e}, 11^{e},\right. \\
\left.12^{e}, 13^{e}, 18^{e}, 19^{e}\right)\end{array}$ & 9 & 260000 & 16,5 & 14,8 & 81 & 19 & 0 & 77 & 45 \\
\hline Total Paris & 100 & 820000 & 19,1 & 7,0 & 66 & 30 & 4 & 63 & 40 \\
\hline
\end{tabular}

Source : Ministère de la Culture et de la Communication, DEPS, 2010

6. Y compris petite et grande couronne parisienne.

7. Parmi tous les arrondissements, les diffuseurs parisiens d'art actuel sont très nettement implantés dans le $6^{\mathrm{e}}(27 \%)$, le $3^{\mathrm{e}}(17 \%)$ et le $8^{\mathrm{e}}(16 \%)$ arrondissements.

8. Françoise Benhamou, Nathalie Moureau et Dominique Sagot-Duvauroux, les Galeries d'art contemporain en France. Portrait et enjeux dans un marché mondialisé, Paris, Ministère de la Culture et de la Communication, DEPS/La Documentation française, coll. « Questions de culture », 2001. 
L'écart de chiffre d'affaires annuel moyen observé entre les structures parisiennes et celles situées hors Paris correspond au choix du statut juridique. Les associations sont celles dont le chiffre d'affaires est le plus faible; elles sont aussi celles que l'on retrouve le plus en région ( $93 \%$ des associations) : à l'opposé, Paris regroupe la totalité des SAS (100\%), les trois quarts des SA et des SNC.

\section{Les diffuseurs hors Paris 9}

Des profils contrastés se dessinent entre structures parisiennes et structures situées en région, plus jeunes et plus fragiles $^{10}$.

En région, les diffuseurs d'art actuel sont localisés dans les grandes villes et dans les lieux touristiques. Si, en RhôneAlpes, les diffuseurs sont concentrés dans les plus grandes villes, Lyon (43\%) puis Grenoble (9\%), en Provence-AlpesCôte d'Azur, ce sont les villes touristiques qui ont leurs faveurs : Saint-Paul-de-Vence (16\%), Nice (15\%), Marseille (10\%), Cannes (5\%) et Saint-Rémy-de-Provence (5\%). Le phénomène est similaire en Bretagne : les villes touristiques de Dinard, Dinan, Pont-Aven et Saint-Malo accueillent davantage de diffuseurs que celles de Brest, Rennes et Quimper.

Le chiffre d'affaires moyen des diffuseurs hors Paris est 5,6 fois moins élevé que celui de leurs confrères parisiens. Quelle que soit la région, le chiffre d'affaires annuel moyen est inférieur à 159000 euros, à l'exception de la Basse-Normandie qui présente un profil particulier ${ }^{11}$.

Plusieurs facteurs expliquent les différences observées entre Paris et les régions.

Tout d'abord, l'âge des structures situées en région est moins élevé que celui de leurs homologues parisiennes. Si l'âge moyen des structures de région est de 12,5 ans, environ un quart d'entre elles $(25 \%)$ existent depuis cinq ans ou moins. Les structures plus anciennes se situent dans le NordPas-de-Calais (19,6 ans), plus anciennes d'ailleurs que les structures parisiennes (19,1 ans), les plus jeunes dans les Pays de la Loire et en Aquitaine (9,9 ans et 10,6 ans respectivement). Toutefois, contrairement à ce qui a été observé à Paris, il n'apparaît pas de corrélation entre l'ancienneté de la structure et son chiffre d'affaires : dans le Nord-Pas-de-Calais par exemple, les structures demeurent fragiles puisqu'aucune d'entre elles n'atteint le seuil de 250000 euros de chiffre d'affaires, tandis que dans les Pays de la Loire, $22 \%$ des structures réalisent un chiffre d'affaires annuel moyen supérieur à 250000 euros.

Les structures de région concentrent davantage leur activité sur des artistes vivants : $82 \%$ des diffuseurs en région réalisent exclusivement leurs ventes à partir d'œuvres d'artistes vivants, contre $66 \%$ des diffuseurs parisiens. De ce point de vue, les diffuseurs semblent courir un risque plus grand en vendant les œuvres d'artistes dont le cercle de collectionneurs reste souvent à construire. Le nombre moyen d'artistes vivants rémunérés par les structures est plus élevé en région (9,9 artistes en moyenne) qu'à Paris (7), en particulier en petite et grande couronne $(14,4)$, en Haute-Normandie (14) et dans les Pays de la Loire (12).

Les régions dans lesquelles les diffuseurs d'art actuel rémunèrent une forte proportion d'artistes de la région sont la Bretagne ( $57 \%$ des artistes rémunérés sont de la même région que leur diffuseur) et le Languedoc-Roussillon (56\%), à l'opposé de la Bourgogne $(26 \%)$ et de la Basse-Normandie $(24 \%)$.

Tableau 2 - Caractéristiques des diffuseurs par territoire (moyennes établies sur trois années d'exercice)

\begin{tabular}{|c|c|c|c|c|c|c|c|c|c|}
\hline Territoire & Effectifs & $\begin{array}{c}\text { CA } \\
\text { moyen } \\
(e n €)\end{array}$ & $\begin{array}{c}\text { Âge } \\
\text { moyen } \\
\text { (années) }\end{array}$ & $\begin{array}{c}\text { Nombre } \\
\text { d'artistes } \\
\text { français } \\
\text { vivants } \\
\text { par structure }\end{array}$ & $\begin{array}{l}\text { Artistes } \\
\text { vivants }\end{array}$ & $\begin{array}{c}\text { Artistes } \\
\text { vivants } \\
\text { et décédés }\end{array}$ & $\begin{array}{l}\text { Artistes } \\
\text { décédés }\end{array}$ & $\begin{array}{l}\text { Artistes } \\
\text { locaux }\end{array}$ & $\begin{array}{l}\text { Artistes } \\
\text { réguliers }\end{array}$ \\
\hline Paris & 37 & 820000 & 19,1 & 7,0 & 66 & 30 & 4 & 63 & 40 \\
\hline Petite et grande couronne & 5 & 150000 & 13,7 & 14,4 & 88 & 11 & 1 & 56 & 40 \\
\hline Régions & 58 & 145472 & 12,5 & 9,9 & 82 & 15 & 3 & 43 & 37 \\
\hline \multicolumn{10}{|l|}{ Dont: } \\
\hline PACA & 14 & 158664 & 11,7 & 10,2 & 83 & 14 & 3 & 34 & 30 \\
\hline Rhône-Alpes & 7 & 123753 & 12,8 & 8,4 & 83 & 16 & 1 & 46 & 31 \\
\hline Bretagne & 5 & 94702 & 11,5 & 9,4 & 75 & 25 & 0 & 57 & 50 \\
\hline Aquitaine & 5 & 155871 & 10,6 & 11,8 & 80 & 17 & 3 & 33 & 47 \\
\hline Midi-Pyrénées & 3 & 115145 & 12,8 & 7,3 & 85 & 13 & 2 & 45 & 25 \\
\hline Basse-Normandie & 3 & 507568 & 14,1 & 10,3 & 80 & 15 & 5 & 24 & 43 \\
\hline Pays de la Loire & 3 & 90459 & 9,9 & 12,0 & 89 & 3 & 9 & 37 & 29 \\
\hline Languedoc-Roussillon & 3 & 79606 & 12,6 & 11,6 & 77 & 23 & 0 & 56 & 45 \\
\hline Bourgogne & 3 & 126702 & 11,3 & 6,1 & 84 & 13 & 3 & 26 & 28 \\
\hline Nord-Pas-de-Calais & 2 & 79585 & 19,6 & 9,6 & 93 & 3 & 3 & 38 & 26 \\
\hline Haute-Normandie & 2 & 96699 & 15,0 & 14,0 & 93 & 0 & 7 & 53 & 35 \\
\hline Autres régions & 8 & 114625 & 14,0 & 10,2 & 83 & 14 & 3 & 34 & 30 \\
\hline Ensemble & 100 & 404681 & 15,0 & 9,0 & 76 & 20 & 3 & 52 & 39 \\
\hline
\end{tabular}

9. Les diffuseurs de la petite et grande couronne parisienne sont intégrés à cette analyse car leurs caractéristiques les rapprochent plutôt des structures situées en région que de leurs consœurs parisiennes.

10. Ces profils incarnent des moyennes qui ne doivent pas faire oublier la forte diversité des situations dans chacun des deux groupes.

11. Une galerie située à Honfleur gonfle à elle seule considérablement le chiffre d'affaires moyen des structures de la région. 
En région, à la différence des structures parisiennes, s'appuyer sur un noyau dur d'artistes n'induit pas nécessairement un chiffre d'affaires élevé. Ainsi, pour bon nombre de structures, constituer un noyau dur d'artistes n'est pas forcément une stratégie commerciale efficace, mais relève davantage d'un choix de politique de valorisation.

\section{VERS UNE TYPOLOGIE ÉCONOMIQUE DES DIFFUSEURS D'ART ACTUEL}

La corrélation entre le montant du chiffre d'affaires, le statut juridique adopté par la structure de diffusion et sa localisation est particulièrement déterminante. La combinaison de ces trois variables permet de dresser une typologie des diffuseurs d'art actuel proposant trois profils distincts (tableau 3) : les associations, les commerçants d'art et les « sociétés commerciales mixtes ».

\section{Les associations}

Les associations sont toutes des galeries. Elles sont peu nombreuses (5\% des diffuseurs d'art actuel résidant fiscalement en France) et très majoritairement localisées en région (92\% d'entre elles). Leur situation est particulièrement précaire : leur chiffre d'affaires annuel moyen est bien inférieur à celui des autres catégories (62 500 euros) et elles sont plus jeunes (11,4 ans en moyenne).

Plus que les autres structures, les associations travaillent exclusivement avec des artistes français vivants (85\%). Peu d'entre elles représentent à la fois des artistes décédés et des artistes vivants (12\%). Le portefeuille des artistes qu'elles proposent est large : ce sont elles qui rémunèrent le plus grand nombre d'artistes français vivants (11,6 en moyenne), s'intéressant majoritairement aux artistes de leur région $\left(58 \%{ }^{12}\right)$. La rotation des artistes présentés est généralement élevée. Les associations sont les structures qui ont le noyau d'artistes réguliers le plus faible parmi les structures étudiées.

Tableau 3 - Caractéristiques économiques des diffuseurs d'art actuel

\begin{tabular}{|c|c|c|c|c|}
\hline & \multirow[t]{2}{*}{ Associations } & \multirow[t]{2}{*}{ Commerçants d'art } & \multicolumn{2}{|c|}{ Sociétés commerciales mixtes } \\
\hline & & & standards & confirmées \\
\hline $\begin{array}{c}\text { Proportion du total } \\
\text { des diffuseurs }\end{array}$ & $5 \%$ & $35 \%$ & $51 \%$ & $9 \%$ \\
\hline Statut juridique & ASS & EP & SARL, EURL & SA, SAS, SNC \\
\hline Type d'activités & $100 \%$ sont des galeries & $\begin{array}{l}88 \% \text { galeries } \\
10 \% \text { antiquaires- } \\
\text { brocanteurs } \\
2 \% \text { éditeurs d'art }\end{array}$ & $\begin{array}{l}93 \% \text { galeries } \\
5 \% \text { antiquaires- } \\
\text { brocanteurs } \\
2 \% \text { éditeurs d'art }\end{array}$ & $\begin{array}{l}92 \% \text { galeries } \\
2 \% \text { antiquaires } \\
5 \% \text { éditeurs d'art }\end{array}$ \\
\hline Situation géographique & $\begin{array}{l}92 \% \text { région } \\
8 \% \text { Paris }\end{array}$ & $\begin{array}{l}76 \% \text { région } \\
20 \% \text { Paris } \\
4 \% \text { couronne }\end{array}$ & $\begin{array}{l}47 \% \text { région } \\
46 \% \text { Paris } \\
6 \% \text { couronne }\end{array}$ & $\begin{array}{l}16 \% \text { région } \\
75 \% \text { Paris } \\
9 \% \text { couronne }\end{array}$ \\
\hline Ancienneté (en 2006) & $\begin{array}{l}\text { Les plus jeunes } \\
\text { ( } 46 \% \text { ont moins } \\
\text { de } 10 \text { ans, } \\
\text { âge moyen } 11,4 \text { ans) }\end{array}$ & $\begin{array}{l}\text { Moyenne } \\
\text { ( } 40 \% \text { ont moins } \\
\text { de } 10 \text { ans, } \\
\text { âge moyen } 14,1 \text { ans) }\end{array}$ & $\begin{array}{l}\text { Moyenne } \\
\text { ( } 42 \% \text { ont moins } \\
\text { de } 10 \text { ans, } \\
\text { âge moyen } 14,4 \text { ans) }\end{array}$ & $\begin{array}{l}\text { Les plus anciennes } \\
\text { (59\% des SA, SAS, SNC } \\
\text { ont plus de } 20 \text { ans, } \\
\text { âge moyen de } 23,9 \text { ans) }\end{array}$ \\
\hline CA annuel moyen & $\begin{array}{l}\text { Le plus faible: } 62500 € \\
(31 \% \text { ont un CA } \\
<10000 €)\end{array}$ & $\begin{array}{l}\text { Assez faible : } 98500 € \\
(38,3 \% \text { ont un } \mathrm{CA} \\
<25000 €)\end{array}$ & $\begin{array}{l}\text { Moyen : } 460600 € \\
(47 \% \text { ont un CA entre } \\
50000 \text { et } 500000 € \text { ) }\end{array}$ & $\begin{array}{l}\text { Le plus élevé: } \\
1536700 € \\
(54 \% \text { on un CA } \\
>500000 €)\end{array}$ \\
\hline $\begin{array}{l}\text { Provenance des œuvres } \\
\quad \text { vendues }\end{array}$ & $\begin{array}{l}\text { Artistes vivants = } 85 \% \\
\text { Vivants et décédés } \\
=12 \% \\
\text { Décédés = 3\% }\end{array}$ & $\begin{array}{l}\text { Artistes vivants = } 79 \% \\
\text { Vivants et décédés } \\
=16 \% \\
\text { Décédés = } 4 \%\end{array}$ & $\begin{array}{l}\text { Artistes vivants = } 75 \% \\
\text { Vivants et décédés } \\
=22 \% \\
\text { Décédés = } 3 \%\end{array}$ & $\begin{array}{l}\text { Artistes vivants = } 65 \% \\
\text { Vivants et décédés } \\
=35 \% \\
\text { Décédés }=1 \%\end{array}$ \\
\hline $\begin{array}{l}\text { Nombre d'artistes } \\
\text { français vivants } \\
\text { vendus }\end{array}$ & $\begin{array}{l}\text { Le plus élevé } \\
\text { (11,6 en moyenne) }\end{array}$ & $\begin{array}{l}\text { Le plus faible } \\
\text { (7,5 en moyenne) }\end{array}$ & Moyen (9,6 artistes) & $\begin{array}{l}\text { Un peu plus } \\
\text { que la moyenne } \\
\text { (10,4 en moyenne) }\end{array}$ \\
\hline $\begin{array}{c}\text { Somme versée } \\
\text { par artiste }\end{array}$ & La plus faible (3 $700 €)$ & Faible $(4000 €)$ & Moyenne $(12000 €)$ & $\begin{array}{l}\text { La plus importante } \\
(33800 €)\end{array}$ \\
\hline $\begin{array}{l}\text { Proportion d'artistes } \\
\text { régionaux }\end{array}$ & Majoritaire (58 \%) & La plus faible (45\%) & Moyenne (53\%) & La plus élevée (63\%) \\
\hline $\begin{array}{l}\text { Proportion d'artistes } \\
\text { réguliers }\end{array}$ & La moins élevée (20\%) & Moyenne (34\%) & La plus élevée (42\%) & $\begin{array}{l}\text { L'une des plus élevées } \\
(41 \%)\end{array}$ \\
\hline
\end{tabular}

Source : Ministère de la Culture et de la Communication, DEPS, 2010 
Deux profils d'associations se dégagent de l'étude qualitative réalisée en région ${ }^{13}$ : d'un côté, les galeries points de vente (circuit marchand) qui suivent les artistes sur le moyen terme, de l'autre les galeries tremplin (circuit institutionnel) qui offrent une première étape dans le parcours artistique et proposent un relais aux artistes désirant accéder à des réseaux nationaux. Il apparaît alors logique que ces dernières associations aient peu d'artistes réguliers. Les associations dont le chiffre d'affaires est le plus élevé (14\% des associations dont le chiffre d'affaires est supérieur à 500000 euros) sont celles qui ont la proportion d'artistes réguliers la plus forte $(47 \%$ au total) ; a contrario, celles dont le chiffre d'affaires est inférieur à 10000 euros (31\%) sont celles dont la proportion d'artistes réguliers est la plus faible (16\% au total). Ainsi, la corrélation entre le chiffre d'affaires et la proportion d'artistes réguliers est significativement positive pour les associations à l'image de ce que l'on observe pour les autres diffuseurs. En moyenne, les prix des œuvres vendues par les associations se situent dans une fourchette basse, ce qui se traduit par un chiffre d'affaires moyen annuel réduit et des sommes versées aux artistes également faibles.

\section{Les commerçants d'art}

Les commerçants d'art, constitués sous la forme d'entreprises patrimoniales, représentent un peu plus d'un tiers des diffuseurs (environ $35 \%$ ). Ils sont davantage localisés en région (un peu plus des trois quarts). Ces structures pratiquent rarement des prix élevés et leur chiffre d'affaires annuel moyen est relativement faible (à peine 100000 euros).

Leur ancienneté est moyenne, proche de celle des SARL (14,1 ans). Il s'agit essentiellement de galeries. Cette catégorie abrite la plus grande proportion d'antiquaires-brocanteurs $(10 \%)$. Les commerçants d'art sont ceux qui ont le plus recours à la vente d'artistes décédés et le nombre d'artistes français vivants qu'ils rémunèrent est le plus faible $(7,5$ artistes en moyenne); les sommes versées chaque année en moyenne par artiste demeurent peu élevées (un peu moins de 4000 euros).

La proportion d'artistes régionaux reste conséquente (45\%). La proportion d'artistes réguliers est de $34 \%$ et corrélée au chiffre d'affaires.

\section{Les sociétés commerciales mixtes}

Une large majorité de diffuseurs $(60 \%)$ se retrouve sous le statut de sociétés commerciales mixtes, qui partagent fréquemment leur portefeuille d'activités entre la vente d'œuvres d'artistes vivants et celle d'artistes décédés. $85 \%$ d'entre elles (soit $51 \%$ du total des diffuseurs d'art actuel) sont composées de structures que l'on qualifiera de «standards » (SARL et EURL) et $15 \%$ (soit $8 \%$ du total) par des sociétés dont l'identité juridique est l'indice d'une activité économique conséquente (SA, SAS, SNC) et que l'on peut qualifier de « confirmées ».

- Les sociétés commerciales dites « standards » constituent le modèle le plus répandu en région comme à Paris. Ce sont essentiellement des galeries (93\%), plus marginalement des antiquaires-brocanteurs $(5 \%)$ ou des éditeurs d'art (2\%). L'adoption d'un statut juridique à responsabilité limitée et la combinaison d'artistes vivants et d'artistes décédés au sein de leur portefeuille d'activités (pour un quart d'entre elles) leur permettent d'assumer une partie du risque inhérent aux activités artistiques. En outre, le suivi régulier d'artistes leur offre une bonne assise économique. Ces structures sont celles qui, de toutes les catégories, affichent la plus grande proportion d'artistes réguliers (environ $42 \%$ ). Leur chiffre d'affaires annuel moyen s'élève à 460600 euros, et leur ancienneté moyenne est de 14,4 ans. Elles versent en moyenne 12000 euros à chaque artiste. Au cours d'une année, elles rémunèrent 9 à 10 artistes vivants français (9,6 en moyenne), ceux-ci résidant majoritairement dans la région (53\%).

- Les sociétés commerciales dites «confirmées », localisées à Paris pour les trois quarts d'entre elles (75\%), sont peu présentes en région (16\%). S'il s'agit essentiellement de galeries, c'est toutefois dans cette catégorie que l'on retrouve la plus grande proportion d'éditeurs d'art (5\%). Caractérisées par leur longévité (durée moyenne d'activité de 24 ans environ), ces structures bénéficient d'une assise financière solide (54\% d'entre elles déclarent un chiffre d'affaires annuel supérieur à 500000 euros). Plus du tiers combinent dans leur portefeuille d'activités la vente d'œuvres d'artistes français vivants à celle d'artistes décédés. Cette dernière influe positivement sur le chiffre d'affaires : les sociétés dont le chiffre d'affaires est le plus faible sont celles qui se concentrent quasi exclusivement sur le travail d'artistes vivants ; a contrario, les diffuseurs qui valorisent le travail d'artistes vivants et d'artistes décédés réalisent un chiffre d'affaires annuel plus élevé. Le nombre d'artistes français vivants qu'elles rémunèrent est moyen (10,4 en moyenne) mais les sommes versées aux artistes sont les plus importantes de l'ensemble de l'échantillon. La proportion d'artistes régionaux est la plus forte $(63 \%)$ et celle des artistes réguliers est parmi les plus fortes également (41\%).

\section{Conclusion}

Le portrait type du diffuseur d'art actuel donne l'image d'une galerie âgée d'une quinzaine d'années (SARL), localisée en Île-de-France, centrée sur la valorisation d'œuvres d'artistes français vivants et qui n'intègre dans son catalogue que peu d'œuvres d'artistes disparus. Son chiffre d'affaires annuel moyen s'élève à 404700 euros dont plus de la moitié est reversé aux artistes qu'elle représente (12000 euros par artiste). La majorité de ces artistes vivent dans la même région qu'elle. Un peu plus du tiers des ventes d'œuvres d'artistes français vivants se concentrent sur un noyau dur constitué de un à cinq artistes fidèles.

Plus importantes en nombre et en taille économique, les structures parisiennes sont souvent plus anciennes et si l'on peut supposer que leur meilleure assise financière est pour partie liée à leur plus grande ancienneté et à leur capacité à appuyer leur activité sur un noyau d'artistes qu'elles suivent depuis longtemps et dont le marché est assuré, leur position centrale leur permet aussi d'accéder à un marché potentiel plus important, la capitale comptant plus de collectionneurs, d'institutions et d'événements. 


\section{Méthodologie de l'étude}

Les résultats de l'étude se fondent sur l'exploitation des données relatives aux diffuseurs d'art plastique français recensés par la Maison des artistes qui gère les contributions au régime de sécurité sociale des artistes. La part diffuseur versée à cet organisme est proportionnelle au chiffre d'affaires réalisé ou aux commissions perçues sur la vente d'œuvres d'art. Le taux est de $1 \%$ appliqué à $30 \%$ du prix de vente des œuvres ou, le cas échéant, du montant de la commission. La Maison des artistes comptabilise à ce jour 20864 diffuseurs, soit 18622 actifs au titre du $1 \%$ dû sur les rémunérations versées à l'artiste ou aux ayants droit, et 2242 actifs au titre de la vente d'œuvres d'art d'artistes vivants ou morts, français ou étrangers.

N'ont été retenus dans le champ de l'analyse que les diffuseurs d'art actuel qui perçoivent une commission ou achètent à des artistes vivants à fin de revente, et ce pour tout ou partie de leur activité. II s'agit très majoritairement de galeries d'art contemporain dont l'activité est précisément d'exposer, de promouvoir et de vendre de telles œuvres mais aussi, plus marginalement, de commerces de brocantes, d'antiquités, ou encore d'éditeurs d'art, de musées ou centres d'art, à l'exclusion des sociétés de ventes volontaires (svv) et des commerces d'autres biens et services (restaurants, bars...) qui peuvent exposer et vendre de telles œuvres. Ne sont pas non plus prises en compte les associations qui ne font, par exemple, que fournir aux artistes les moyens d'exposer, à titre gratuit ou onéreux. Enfin, les galeries de photographie, ne contribuant pas au régime, ne sont pas non plus prises en compte.

Un ensemble de 516 diffuseurs d'art actuel, représentatif de leur localisation géographique (région) et de leur tranche de chiffre d'affaires déclaré en 2006, a été constitué.

Les données ont été collectées sur la base de la déclaration des diffuseurs pour 2004, 2005 et 2006.

\section{RÉSUMÉ}

L'analyse de l'activité économique des diffuseurs d'art actuel s'appuie sur les déclarations de rémunération et de ventes d'œuvres d'art d'artistes français effectuées par les diffuseurs auprès de la Maison des artistes, organisme gestionnaire des contributions au régime de sécurité sociale des artistes. L'examen du chiffre d'affaires, corrélé à la forme juridique du diffuseur, à l'ancienneté de la structure, à sa localisation géographique et aux liens entretenus avec les artistes qu'elle diffuse, permet de caractériser l'activité de diffusion et de dresser des profils distincts. L'analyse révèle également la concentration de l'activité de diffusion au sein des galeries d'art parisiennes, plus importantes en nombre et en taille économique, et la caractérise.

\section{ABSTRACT}

The analysis of contemporary art dealers' economic activity is based on statements of earnings and sales of French artists' works as declared by dealers registered with the Maison des Artistes, the organisation which manages contributions to the artists' social security system. By examining turnover and correlating it with the dealer's business type, how long they have been in existence, their geographic location and their relationships with the artists whose work they distribute, one can distinguish distribution activity and draw up distinct profiles. This analysis also gives a detailed picture of how concentrated the activity of dealing is from Parisian art galleries, which are economically larger and more numerous.

Tous les documents publiés par le DEPS sont téléchargeables sur http://www.culture.gouv.fr/deps

Le DEPS n'assurant pas de diffusion physique de ces documents, nous vous proposons de vous informer régulièrement des parutions par message électronique. Pour ce faire, merci de bien vouloir nous communiquer votre courriel à l'adresse contact.deps@culture.gouv.fr 